\title{
Genotype-Environment Interaction and Stability of Fiber Properties and Growth Traits in Triploid Hybrid Clones of Populus Tomentosa
}

\author{
Jian Wu \\ Beijing Forestry University \\ Qing Zhou \\ Beijing Forestry University \\ Yaru Sang \\ Beijing Forestry University \\ Xiangyang Kang \\ Beijing Forestry University \\ Pingdong Zhang ( $\square$ zhangpd@bjfu.edu.cn ) \\ Beijing Forestry University https://orcid.org/0000-0001-6006-360X
}

\section{Research article}

Keywords: genotype-environment interactions, stability parameter, clonal repeatability, fiber property, growth trait, Populus tomentosa

Posted Date: December 23rd, 2020

DOI: https://doi.org/10.21203/rs.3.rs-132369/v1

License: (c) (i) This work is licensed under a Creative Commons Attribution 4.0 International License. Read Full License 


\section{Genotype-environment interaction and stability of fiber properties and growth traits in triploid hybrid clones}

\section{of Populus tomentosa}

Jian $\mathrm{Wu}^{1,2,3,4}$, Qing Zhou ${ }^{1,2,3,4}$, Yaru Sang ${ }^{1,2,3,4}$, Xiangyang Kang ${ }^{1,2,3,4}$ and Pingdong Zhang ${ }^{1,2,3,4 *}$

${ }^{1}$ Beijing Advanced Innovation Center for Tree Breeding by Molecular Design, Beijing Forestry University, Beijing 100083, China

${ }^{2}$ National Engineering Laboratory for Tree Breeding, Beijing Forestry University, Beijing 100083, China

${ }^{3}$ Key Laboratory of Genetics and Breeding in Forest Trees and Ornamental Plants, Ministry of Education, Beijing Forestry University, Beijing 100083, China

${ }^{4}$ College of Biological Sciences and Technology, Beijing Forestry University, Beijing 100083, China

* Correspondence: zhangpd@ bjfu.edu.cn

\section{Abstract}

12 Background: Clones provide a sensitive method for evaluating genotypic stability and detecting genotypeenvironment $(\mathrm{G} \times \mathrm{E})$ interactions because of non-additive genetic effects among clones and no genetic effect among ramets of an ortet. The experiments aimed at confirming and expanding the earlier findings, estimating stability parameters, and providing accurate estimates of clonal repeatabilities and genetic gains for an triploid breeding programme of Populus tomentosa Carr.

17 Results: Six 5-year-old clonal trials established in Northern China were used to determine the clonal variation, clone $\times$ site interactions and the stability parameters of fiber properties of wood and growth traits. Three hundred sixty trees from ten hybrid clones were collected in the six sites. The clonal and site effects had a highly significant effect $(P<0.001)$ for all studied traits. The clone $\times$ site interactions had a highly significant effect $(P<0.001)$ on fiber length (FL), coarseness $(\mathrm{C})$, and tree growth (tree height $[\mathrm{H}]$, diameter at breast height $[\mathrm{DBH}]$ and stem volume $[\mathrm{SV}])$, and a moderate effect $(P<0.05)$ on fiber width $(\mathrm{FW})$ and fiber length/width $(\mathrm{FL} / \mathrm{W})$. For FL and SV, most of the triploid hybrid clones had higher reaction norms to the improvement in growth conditions and higher phenotypic plasticity. The estimated clonal repeatability of FW (0.93) was slightly higher than for FL (0.89), FL/W (0.83), C (0.91), DBH (0.76), H (0.85), and SV (0.80). Three clonal testing sites were sufficient to estimate quantitative parameters of fiber properties. However, more than three clonal testing sites will help improve the accuracy of quantitative parameters of growth traits.

28 Conclusions: Our results unraveled that accurate estimation of quantitative parameters for growth traits in triploid hybrid clones of Populus tomentosa required more clonal testing sites than fiber properties.

30 Keywords: genotype-environment interactions; stability parameter; clonal repeatability; fiber property; growth trait; Populus tomentosa

\section{Background}

33 Chinese white poplar, Populus tomentosa Carr. (section Populus, family Salicaceae, genus Populus), is a widely 34 distributed tree species in northern China and generally grows in mixed-species forest with Robinia pseudoacacia L. 
Since the first triploid hybrid of $P$. tomentosa was artificially synthesized in 1998, the triploid hybrid clones has exhibited superior growth in China [1]. The latest research progress in wood fiber have led to the use of short fibers mixed with coniferous long fibers to produce high-quality paper, or even without the long fibers [2]. The Chinese forestry industry has shown a new focus on the using hybrid poplar for manufacturing evenly short fibers. Thus, An obvious increase in Chinese white poplar use is expected, which in turn arouse interest in breeding selected triploid hybrid clones [3-5].

The triploid hybrid clones of $P$. tomentosa have been shown to display a high degree of variability in growth, fiber traits, wood basic density, wood chemical properties [1,3-6]. This variability indicates that intriguing gains may be obtained through selection. The potential advantages of utilizing clonal methods in poplar hybrid clones were reviewed by several authors [7-8]. Genotype $\times$ environment $(\mathrm{G} \times \mathrm{E})$ interaction normally refers to the performance differences of genotypes between environments [9]. Testing and selection in tree improvement programmes are complicated by this interaction, and leading to reduced total genetic gains. In general, the literature on $\mathrm{G} \times \mathrm{E}$ is plentiful for forest trees [10-11]. However, the number of studied clones is relatively limited [12]. It is expected that clones are more interactive in different environments than either seed or families origins, because there is no genetic effects among ramets of an ortet and non-additive genetic effects among clones are substantial. Therefore, Clones can provide a sensitive method for evaluating genotypic stability and detecting $\mathrm{G} \times \mathrm{E}$ interactions [13].

To obtain a better understand the genetic control of growth traits, fiber properties, wood basic density and wood chemical properties, basic genetics such as $\mathrm{G} \times \mathrm{E}$ interactions, inter-trait and inter-site correlations, clonal genetic variation and repeatabilities for triploid hybrid clones of $P$. tomentosa were recently estimated [4-5,14-15]. Both site effects and clonal effects were found to be significant for all tested traits and $G \times E$ interactions were also significant for all studied traits except for fiber length and holocellulose [5,14]. This basic information is crucial to estimate expected genetic gains and develop suitable strategies for clonal forestry. However, most of the studies were conducted on three or four sites, whereas the present study was performed on six sites. For the purpose of tree improvement, accurate estimate of quantitative parameters requires that different genotypes are tested at more locations.

60 The objectives of the present study were: (1) to confirm and expand the earlier findings; (2) to estimate stability 61 parameters of these triploid clones; and (3) to provide accurate estimated values of genetic gains and clonal repeatabilities for improvement program of Chinese white poplar.

\section{Results}

\section{Basic statistics and variation within and between sites}

65 The mean values, standard error (SE) of the mean, ranges of maximum and minimum values, and coefficients of 66 phenotypic variation of all traits in the six clonal trials are presented in Table 3. A fairly obvious difference in growth was found between the six locations, with trees exhibiting the largest growth was observed at the Taiyuan. For fiber properties, the longest fiber length (FL) and the greatest fiber length/width ratio (FL/W) were found at the Gaotang. Trees from the Pinggu site had the shortest FL and the lowest FL/W. The difference between the longest and shortest means of FL was $7.9 \%$ (Table 3). However, the thinnest fibers were observed at Xiangfen, and the thickest, at 71 Pinggu. The combined analysis of all six trials displayed that all studied traits had significant site effects (Table 4). 
FL, fiber width (FW), and FL/W showed a small phenotypic variation $(\mathrm{CV}=3.2-5.5 \%)$ and the values were much lower than those of coarseness $(\mathrm{C})$, diameter at breast height (DBH; at $1.3 \mathrm{~m}$ above ground level), tree height $(\mathrm{H})$, and stem volume (SV).

\section{Clonal variation and repeatability}

The results of the analysis of variance for the combined six sites are presented in Table 4. Significant clonal effects for all tested traits were been seen (Table 4). For all studied traits, the variance caused by error (That is, the differences between ramets within a clone within a site) accounted for most of the variation, accounting for 48.4 to $75.4 \%$ of the overall variation. However, most of the variance in FW (35.7 \%) was caused by the clone. Hence, the highest estimated repeatability of clone mean $(0.93)$ and the estimated individual-tree clonal repeatability $(0.36)$ were observed for the FW.

The estimated clonal repeatability and estimated individual-tree clonal repeatability of all the studied traits at the each site are shown in Table 5. No significant differences were found between the clones for H, DBH, and SV at the Yanzhou sites, FL/W at the Gaotong sites, as well as FW and FL/W at the Pinggu sites. Therefore, we did not estimate the clonal repeatability for the six traits. The estimated clonal repeatability varied from 0.73 to 0.90 for FL, from 0.68 to 0.95 for $\mathrm{FW}$, from 0.54 to 0.90 for $\mathrm{FL} / \mathrm{W}$, and from 0.78 to 0.93 for $\mathrm{C}$. The estimated clonal repeatability of the growth traits ranged from 0.58 to 0.90 (Table 5). However, these differences in estimated clonal repeatability were often non-significant.

\section{Clone $\times$ site interaction, stability, and reaction norms of triploid hybrid clones}

In this study, all fiber properties and growth traits were observed to have significant interaction of clone $\times$ site (Table 4). The level of significance for FW (0.031) and FL/W (0.014) was moderate and a highly significant effect $(P<$ 0.001) was observed for FL, C and tree growth. Higher estimated ratio (57.9\%) in $\mathrm{H}$ of clone $\times$ site interaction variance component to the total variance component of clonexsite interaction plus clone were observed than the other traits.

The intersite (B-type) genotypic correlations are shown in Table 6. Some intersite genotypic correlations between the same traits were not estimated because no significant differences were observed between the clones for $\mathrm{H}, \mathrm{DBH}$, and SV at the Yanzhou site, FL/W at the Gaotong site, and FW and FL/W at the Pinggu site. Most of the intersite genotypic correlations between the same traits at different sites were either moderate or strong, except for $\mathrm{H}$ at Zhengzhou-Taiyuan and FL at Pinggu-Taiyuan (Table 6). For FL, FL/W, and the growth traits, the correlations were lower when the Taiyuan site was included.

The stability parameters for FL and SV of the individual triploid hybrid clones across the six clonal trials, including the Finlay-Wilkinson parameters and the Shukla stability variances that characterize the reaction norms of the clones are shown in Table 7. The stability parameters of the all studied clones were estimated only for FL and SV. For FL, the Shukla's stability of clones B330 and B331 was slightly higher than those of the other clones due to their lower stability variance (1.01 x $10^{-4}$ and $2.28 \times 10^{-4}$, respectively). However, for SV, lower stability variances were observed in clones B302, B303, and B306. Therefore, clones B302, B303, and B306 had higher stability for SV than 
B304, and B306 varied from 1.214 to 1.429, indicating that the four clones had higher reaction norms. However, the clone, B304, B330, and B331 had higher reaction norms for SV because their regression coefficients $\left(b_{i}\right)$ were $1.315,1.411$, and 1.477 , respectively.

\section{Inter-trait genetic correlations, correlated genetic response, and genetic gain}

112 The genotypic and phenotypic correlations between all the studied traits at individual sites are presented in Table 8.

113 At all sites, a positive estimated correlation was found between FL and growth traits. A significant positive estimated

114 genetic correlation between FW and $\mathrm{C}$ was also observed at each site. However, a negative estimated genotypic

115 correlation between growth traits (except for $\mathrm{H}$ ) and the $\mathrm{C}$ existed. The results suggested that selection for growth 116 traits (except for $\mathrm{H}$ ) might lead to a slight decrease in $\mathrm{C}$. SV, H, and DBH had some strong positive autocorrelations 117 since $\mathrm{SV}$ was derived from $\mathrm{H}$ and $\mathrm{DBH}$.

118 The predicted genetic gains of the hybrid clones at the six sites for different selection are presented in Table 9.

119 The results are based on the assumption of the estimated repeatabilities and correlations from direct clonal selection 120 and the related genetic responses in all the studied traits. The predicted genetic gains were identical in $\mathrm{C}$ for Pinggu 121 and Zhengzhou (6.1\%), while the Taiyuan site had the highest gain (8.4\%). However, the gains in $\mathrm{C}$ were about 122 twice the gains observed for FL. The selection for $\mathrm{C}$ led to the equal gains in FW as direct selection for FW. The 123 selection for DBH led to the gain in SV equal to that of the direct selection for SV but resulted in a slight decrease in $124 \mathrm{C}$ (Table 9). Among growth traits, selection for $\mathrm{C}$ resulted in the lowest gains in DBH and SV. However, it had a 125 greater negative impact on SV.

\section{Discussion}

127 Site effects represent the response of trees to the combined influences of edaphic and regional climatic conditions 128 [16]. Even though the current trials were not designed to seperate these different effects, some conclusions can still 129 be drawn [16]. The poor field performance in growth at Yanzhou was may be owing to poor drainage (due to soil 130 compression) and wet weather at early stages of the experiment. Significant site effects were observed in the 131 combined analysis of all traits (Table 4). Moreover, a positive correlation between SV and latitude and negative 132 correlations between SV and longitude, as well as rainfall and mean annual temperature, were observed (Fig. 1.), 133 suggesting that edaphic and regional climatic conditions had significant effects on the growth traits. Our findings 134 agreed with the results of former studies of triploid hybrid clones of P. tomentosa [4-5], poplar hybrids [16-17], and 135 P. tremuloides Michx. [18]. At all sites, the fiber properties of the triploid hybrid clones had less phenotypic 136 plasticity than the growth traits, as evidenced by the lower CV values, indicating that site effects were relatively 137 lower for fiber properties than growth traits.

138 Former studies recorded that the effect of clone $\times$ site interactions on the growth traits at three clonal trials was 139 greater than the clonal effect [4-5]. Randall and Cooper [19] reported that the complexity of testing increased due to 140 such a high interaction. If the correlations between test and planting environments were alike and the objectives of 141 an improvement program were restricted enough to allow selection for certain types of locations, then these 142 interactions may be utilized. The effect of clonal effects on the growth traits in this study was, on the contrary, 143 greater than the clone $\times$ site interactions when the number of testing sites was six. This suggested that an increase in 
the number of clonal testing sites help improve the accuracy of clonal effects, thus reducing the complexity of clonal testing.

146 It seems that most selected triploid hybrid clones of $P$. tomentosa will probably be exploited on a relatively wide 147 range of sites. In this situation, the effectiveness of selection will be greatly reduced because of large clone $\times$ site 148 interactions unless performance is measured on a relatively large number of sites. Additionally, this interaction will 149 probably be decreased when the region split into planting zones according to the type of terrain. An increase in gain 150 from clonal selection within each site type will be expected. This interaction may be, also, decreased by using stable 151 clones. The gain from a decrease in clone $\times$ site interaction must be compared with the loss from eliminating clones 152 characterized with high growth potential.

153 Zhang et al. [5] reported that the clone $\times$ site interactions were significant for growth traits and fiber properties in triploid hybrid clones of $P$. tomentosa except for FL. In this study, the clone $\times$ site interactions had a highly significant effect on all the studied traits, indicating that triploid hybrid clones of $P$. tomentos $a$ exhibited various relative performances on the six sites. The ratio of the variance component of clone $\times$ site interaction and clone is 1.4 for H, 0.5 for DBH and 0.9 for SV. In an example recorded by Lindgren [20], the ratio ranged from 0 to 3, and in most cases it was lower than 1 .

In this study, no significant positive type B genotypic correlations between genotypic values at Xiangfen-Taiyuan sites for all studied traits were observed (Table 6), which suggested that a true $\mathrm{G} \times \mathrm{E}$ interaction existed. The differences between these sites may be contributed to the clone $\times$ site interaction. However, the type $\mathrm{B}$ genotypic correlations for FL and C between the sites were mostly positive (Table 6), which indicated that the fiber properties were more stable than the growth traits. This result corresponded to the findings of Yu et al. [21] that the relative performance of genotypes in different environments with regard to fiber traits was quite stable.

In forest tree breeding, it is crucial to predict the yield of a genotype in different environments. The relative performances in stability analysis are determined by regression coefficient. This information can contribute to develop suitable genotypes for specific environments in forest tree breeding. However, this information is irrelevant if all the tested environments are in one planting region, and each represents the same scale of zone to be planted. Selection for the total mean is all, nonetheless, that is necessary to ensure the highest total gains.

170 For forest tree breeding, what is more important is to predict yield of a genotype in different environments. This concept of stability may be determined by the stability variances [22] and the regression coefficients [23]. In this 172 study, the results indicated that each of the studied clones had various magnitudes of phenotypic plasticity and 173 specific patterns of FL and SV at the six sites. One hypothesis considers that genetic variation and plasticity 174 represent alternative strategies for dealing with environmental heterogeneity [24]. However, other hypotheses 175 believe that a positively correlated between phenotypic plasticity and genetic variation [25]. Higher sensitivity 176 regarding changes in the FL was found for clone B305 and B306 because they had high Shukla's stability variances. 177 However, the regression coefficients $\left(b_{i}\right)$ of clones B302, B303, B304, and B306 for FL ranged from 1.214 to 1.429, 178 suggesting that the four clones had higher reaction norms than the other clones. The stability values for the FL of the 179 triploid hybrid clones were different for the different methods. This could be partially explained by the different 180 principles that are used to estimate the stability parameters. Similar results were found for temporal stability of vigor 
in rubber by Gouvêa et al. [26] and for wheat yield by Mohammadi and Amri [27]. High values of the Finlay-

182 Wilkinson stability parameter, i.e., the regression coefficient of the clone values on location value, determine the clones that benefit from a productive location, while high intercept coefficients determine the clones that can grow well under limited resource conditions. As shown by the higher regression coefficient means of clone B304, B330, and B331 for SV, these clones have higher norms of reaction to the improvement in growth environments and higher phenotypic plasticity.

Generally, an increase in tree growth was related to a slightly higher FL. A number of previous studies have shown that FL increased as the increasing tree growth [28-30]. For example, Xie et al. [31] reported that a significant positive estimated genetic correlation between FL and growth traits was observed in Populus euramericana (Dode) Guinier. In the present study, the genetic correlations between growth traits and FL were also positive at the six sites, indicating that triploid breeding of Populus not only improved the FL, but also increased the tree growth.

Clonal repeatability of the fiber properties and growth traits across all six sites were medium to high (Table 4). For fiber properties, the estimated clonal repeatabilities ranged from 0.83 to 0.93 , which was consistent with those that has been recorded in the literature [4-5,32]. This suggested that three clonal testing sites were enough to estimate quantitative parameters for fiber properties. However, clonal repeatabilities were estimated to be 0.76 for $\mathrm{H}$, 0.85 for DBH and 0.80 for SV, which were slightly higher than those estimated at three clonal trails [5]. This implied that increasing the number of testing sites help improve the accuracy of clonal repeatabilities of growth traits.

The analyses of the present study were based on nine genotypes and a relative small number of testing sites measured over only five years. Therefore, The results from this study should be further verified by subsequent larger and longer trials. The implication of the $\mathrm{G} \times \mathrm{E}$ interaction for estimating the gain of genetically improved triploid hybrid clones of $P$. tomentosa should be further studied.

\section{Conclusions}

The experiments and analyses lead to the conclusion that the clone and site had a highly significant effect $(P<0.001)$ for all studied traits. The clone $\times$ site interactions had a moderate effect on FW and FL/W, and a highly significant effect $(P<0.001)$ on FL, C, and tree growth. The stability parameter analysis showed that most of the triploid clones had higher reaction norms to the improvement in growth conditions and higher phenotypic plasticity. The estimated clonal repeatability of FW (0.93) was slightly higher than for C (0.91), FL (0.89), FL/W (0.83), H (0.85), DBH (0.76), and SV (0.80). Three clonal testing sites were good enough to estimate quantitative parameters of fiber properties. However, more than three clonal testing sites will help improve the accuracy of quantitative parameters of growth traits. The findings in this study were due to a certain number of triploid hybrid clones derived from several parents $(n=5)$. Therefore, the implication of $G \times E$ interaction for calculating the gain of genetically improved triploid hybrid clones of P. tomentosa should be further investigated.

\section{Methods}

\section{Designing of experiments}


216 In the present study, the materials used were sampled from six triploid hybrid poplar clonal trials in northern China.

217 These trials were established by Beijing Forestry University using cuttings of $P$. tomentosa on sandy loam with

218 typical soil fertility. The clonal trials at Yanzhou, Gaotang and Xiangfen were built in the spring of 2004 and the

219 clonal trials at Pinggu, Taiyuan and Zhengzhou were established in the spring of 2005. The characteristics of the six

220 clonal experiments are shown in Table 1 . The planted cuttings were $1.5-2.0 \mathrm{~cm}$ in diameter and $20-25 \mathrm{~cm}$ in length.

221 The experiments were repeated three times in the nursery. The experiments included one diploid clone and nine

222 triploid hybrid clones at each site (Table 2). The nine triploid hybrid clones were preselected among the triploids

223 derived from five parent trees because they exhibited good growth rate [33]. The diploid clone (M1319) was an elite

224 tree of $P$. tomentosa which grows faster. A randomized complete block design with three replications was used in

225 each clonal trial (240 trees per plot, resulting in a total of 720 trees per clone per trial). Ten clones were planted on

226 the rectangular plots at each location, and each plot contained 240 trees $(4 \times 60$ trees $)$ with $2 \mathrm{~m} \times 3 \mathrm{~m}$ spacing. No

227 thinning was conducted during the testing period.

\section{Measurement of fiber properties and growth traits}

229 A total of 360 trees were randomly selected from the six trials sites. Trees were collected randomly, 6 ramets (the 6

230 trees) each clone were sampled fully at random on per location. In the triploid poplar breeding program of Beijing

231 Forestry University, the overall $\mathrm{H}$ and DBH of all tree samples were measured by workers at the age of 5 years. The

232 SV of each sample was estimated according to the volume function of DBH and H used by Chen [34]. All sampled

233 trees were shipped to Beijing Forestry University. For laboratory measurements, a 10-cm-thick stem disk was

234 harvested from the breast height of each sample. Matchstick-sized wood samples (each sample implying two pairs of

235 annual rings) were cut from the stem disks to evaluate the fiber properties, and then immersed in a boiling 1:1 (v/v)

236 mixed solution of hydrogen peroxide and acetic acid. Afterwards, FL, FW, and C was measured according to the

237 methods described by Zhang et al. [4] using the Lorentzen \& Wettre (LW) Fiber Tester (AB Lorentzen \& Wettre,

238 Kista, Sweden).

\section{Statistical analysis}

240 When the main statistical analysis and the estimation of genetic statistics were conducted, the diploid clone (M1319)

241 was not included. Thus, only the nine triploid clones took part in the main variance analysis and estimates of genetic

242 parameters. Analyses of variance were performed according to the UNIVARIATE program of the SPSS software

243 (SPSS for Windows, version 13, SPSS, Chicago, IL). Analysis of variance was used to analyze the variation between

244 the ramets of the sample clones at a location based on the following linear model (Equation (1)):

$$
X_{i k}=\mu+C_{i}+\varepsilon_{i k},
$$

246 where $X_{i k}$ represents the observation of the $k$ th ramet of the $i$ th clone; $\mu$ represents the general mean; $\mathrm{C}_{i}$ represents the

247 effect attributed to the $i$ th clone and $\varepsilon_{i k}$ represents random error. The clone $\times$ replicate effects were not included in the

248 linear model (Equation (1)) as the six sampled trees per clone per location were randomly selected.

249 The clonal repeatabilities were calculated within a location as: 


$$
R_{c}^{2}=\frac{\hat{\sigma}_{c}^{2}}{\hat{\sigma}_{c}^{2}+\frac{\hat{\sigma}_{e}^{2}}{k}}
$$

and the clonal repeatability of the individual-tree $\left(R_{b}^{2}\right)$ was estimated according to the following equation:

$$
R_{b}^{2}=\hat{\sigma}_{c}^{2} /\left(\hat{\sigma}_{c}^{2}+\hat{\sigma}_{e}^{2}\right),
$$

where $k$ is the average value of tree samples per clone within a location; $\hat{\sigma}_{c}^{2}$ is the estimated variance of clone; $\hat{\sigma}_{e}^{2}$ is 254 the variance among the ramets within the clones.

The six locations were jointly analyzed using the following linear model [17] (Equation (4)):

$$
X_{i j k}=\mu+C_{i}+L_{j}+C_{i} L_{j}+\varepsilon_{i j k}
$$

where $X_{i j k}$ represents the performance of the $k$ th ramet of the $i$ th clone within the $j$ th location; $\mu$ represents the general mean; $C_{i}$ represents the effect attributed to the $i$ th clone; $L_{j}$ represents the effect attributed to the $j$ th location; $C_{i} L_{j}$ represents the interaction between the $i$ th clone and $j$ th location, and $\varepsilon_{i j k}$ represents random error. Only the location was regarded as a fixed effect and all other terms were regarded as random effect.

The clonal repeatabilities were estimated using the equation:

$$
R_{c}^{2}=\frac{\hat{\sigma}_{c}^{2}}{\frac{k_{2} \hat{\sigma}_{c}^{2}}{k_{2}}+\frac{k_{1} \hat{\sigma}_{L \times c}^{2}}{k_{2}}+\frac{\hat{\sigma}_{e}^{2}}{k_{2}}}
$$

and the clonal repeatability of individual-tree was calculated across locations according to the following equation:

$$
R_{b}^{2}=\hat{\sigma}_{c}^{2} /\left(\hat{\sigma}_{c}^{2}+\hat{\sigma}_{L \times c}^{2}+\hat{\sigma}_{e}^{2}\right),
$$

where $k_{1}$ is the coefficient related to the variance caused by the clone $\times$ site interaction item $\left(\hat{\sigma}_{L \times c}^{2}\right) ; k_{2}$ is the coefficient related to the variance caused by the clonal variation $\left(\hat{\sigma}_{c}^{2}\right)$. The standard errors (SE) for the estimates of clonal repeatability were estimated according to the following equation [35]:

$$
\operatorname{SE}\left(R_{c}^{2}\right)=\sqrt{\frac{2\left(1-R_{c}^{2}\right)^{2}\left[1+\left(k_{2}-1\right) R_{c}^{2}\right]^{2}}{k_{2}\left(k_{2}-1\right)(N-1)}},
$$

where $N$ is the number of tested clones. The SE for the clonal repeatability estimates of individual-tree were also estimated according to the Equation (7), where $R_{b}^{2}$ was used instead of $R_{c}^{2}$.

The genetic correlation coefficients $\left(r_{A(X Y)}\right)$ between traits at a location were computed using the equation [36]:

$$
r_{A(X, Y)}=\frac{\hat{\sigma}_{c(x, y)}}{\sqrt{\hat{\sigma}_{c(x)}^{2} \hat{\sigma}_{c(y)}^{2}}},
$$

where $\hat{\sigma}_{c(x, y)}$ is the clonal component of covariance estimated among traits $x$ and $y ; \hat{\sigma}_{c(x)}^{2}$ is the clonal variance component estimated for trait $\mathrm{x} ; \hat{\sigma}_{c(\mathrm{y})}^{2}$ is the clonal variance component estimated for trait $\mathrm{y}$. Similar to using the 275 mean squares for estimating variance components, the cross-products were used to estimate the corresponding clone component of variance from the data collected from the same individual tree.

277 The approximate SE of genetic correlation estimates were estimated based on the following formula [9]: 
where $r$ is the estimate of genetic correlation; $R_{x}^{2}$ is the estimate of the clonal repeatability of the trait $x$; $R_{y}^{2}$ is the estimate of the clonal repeatability of the trait $y ; \hat{\sigma}_{\left(R_{x}^{2}\right)}$ and $\hat{\sigma}_{\left(R_{y}^{2}\right)}$ are the standard error for $R_{x}^{2}$ and $R_{y}^{2}$, respectively. Using Equation (7) as the basis for the $t$-tests, the significance of the genetic correlations between the trait was tested.

The genetic correlation estimates among the same traits for pairs of locations were estimated to assess the contribution of each pair of treatments to the overall clone $\times$ environment. These type B genotypic correlations were computed according to the measurements of sample ramets at different locations from the same clones planted using the equation [37]:

$$
r_{B(X, Y)}=\frac{r_{P(x 1, y 2)}}{R_{C(x 1)} R_{C(y 2)}}
$$

where $r_{p(x l, y 2)}$ is the coefficient of phenotypic correlation between the clonal means estimated between $x$ measured at location 1 and $y$ measured at location $2 ; R_{c(x 1)}$ and $R_{c(y 2)}$ are the square roots of the mean repeatability of the clones $\mathrm{x}$ and y estimated at location 1 and 2 , respectively.

The SPSS PROC CORR software was used to calculate the phenotypic correlations of the clonal means for each pair of sites, and the significance test was performed using the $F$-test.

In the present study, it is assumed that the selection intensity is equal to 1.271 , which corresponds to selecting four clones from 18 or three clones from 14 [16]. According to the relevant response of the clonal selection in trait $x$, the expected genetic gain in trait $y \Delta G_{y}$ can be predicted using the formula [9]:

$$
\Delta G_{y}=i_{x} \sqrt{R_{x}^{2}} \sigma_{y} r_{x y}
$$

where $i_{x}\left(i_{x}=1.271\right)$ is the selection intensity; $R_{x}^{2}$ is the clonal repeatabilities for trait $x$; $\sigma_{y}$ is the standard deviation of clonal for trait $y ; r_{x y}$ is the genetic correlation coefficients between trait $x$ and $y\left(r_{x y} \leq 1\right)$.

\section{Estimation of stability parameters}

To evaluate the stability of the individual clones across the six experimental locations, the stability variances were estimated using the Shukla model [22], where lower values indicate higher stability, and the significance test was conducted using the $F$-test. Subsequently, the stability of the individual clones across locations was also estimated using the Finlay and Wilkinson stability parameter [23]. The triploid clones were grouped according to the size of their regression coefficients $\left(b_{i}\right)$, less than, equal to, or $>1$. Clones with $b_{i}>1$ are more adapted to favorable growing conditions, those with $b_{i}<1$ are adapted to unfavorable environmental conditions, and those with $b_{i}$ equal to 1 have an average adaptation to all environments [23].

\section{Abbreviations}


CV $p$ \%: coefficients of phenotypic variation; FL: fiber length; FW: fiber width; FL/W: fiber length/width; C: coarseness; H: tree height; DBH: diameter at breast height; SV: stem volume; $R_{b}^{2}$ : repeatability of individual-tree

310 clonal; $R_{c}^{2}$ : repeatability of clonal means; SE: standard errors; YZ: Yanzhou; GT: Gaotang; XF: Xiangfen; PG:

311 Pinggu; ZZ: Zhengzhou; TY: Taiyuan; $F$ : Fisher's criteria; $P$ : probability levels; $t$ : Student's criteria; $b_{i}$ : regression

312 coefficient

313 Declarations

314 Ethics approval and consent to participate

315 Not applicable.

316 Consent for publication

317 Not applicable.

318 Availability of data and materials

319 All data generated or analysed during this study are included in this published article.

320 Competing interests

321 The authors declare that they have no competing interests.

$322 \quad$ Funding

323 This study was mainly supported by the $13^{\text {th }}$ National Key Research and Development Program of China 324 (2016YFD0600403).

\section{Authors' contributions}

326 JW was responsible for generating the phenotypic data set, analyzing the results, and writing the paper. QZ and YS

327 was responsible for tree growth measurements. XK was responsible for planning and guiding this research. PZ was

328 responsible for planing and building the clonal trails, collecting wood discs sample, fiber properties measurements 329 and revised the paper. All authors have read and approved the manuscript.

\section{$330 \quad$ Acknowledgements}

331 The authors would like to thank Dr. Jicheng Pei from Tianjin University of Science and Technology for his 332 assistance with fiber properties measurements.

\section{References}

334 1. Zhu ZT, Kang XY, Zhang ZY. Studies on selection of natural triploids of Populus tomentosa. Sci Silvae Sin. 335 1998;34:22-31.

336 2. Ranua J. Why is the Finnish forest industry interested in aspen? Sorbifolia. 1996;4:178-180.

337 3. Wu F, Zhang PD, Pei JC, Kang XY. Genetic variation of fiber properties of triploid Chinese white poplar planted 338 in Beijing. J Northeast For Univ. 2011;39:4-7. 
4. Zhang PD, Wu F, Kang XY. Genotypic variation in wood properties and growth traits of triploid hybrid clones of Populus tomentosa at three clonal trials. Tree Genet Genom. 2012;8:1041-1050. https://doi.org/10.1007/s11295012-0484-9.

5. Zhang PD, Wu F, Kang XY. Genetic control of fiber properties and growth in triploid hybrid clones of Populus tomentosa. Scand J For Res. 2013;28:621-630. https://doi.org/10.1080/02827581.2013.829868.

6. Pu JW, Song JL, Rao CL. Studies on variations of chemical components of triploid clones in Populus tomentosa Carr. Paper Sci Technol. 2002;21:1-4.

7. Michler CH, Bauer EO. High frequency somatic embryogenesis from leaf tissue of Populus spp. Plant Sci. 1991;77:111-118. https://doi.org/10.1016/0168-9452(91)90186-C.

8. Yu QB. Can physiological and anatomical characters be used for selecting high yielding hybrid aspen clone? Silva Fennica. 2001;35:137-146. https://doi.org/10.14214/sf.591.

350 9. Falconer DS. Introduction to quantitative genetics, 3rd ed. London: Longman; 1981. p. 285-286.

10. SkrØppa T. A critical evaluation of methods available to estimate the genotype $\times$ environment interaction. In: Proceedings of the Genotype $\times$ Environment Interaction. Sweden: Nordic Group of Forest Tree Breeders; 1982. p. 23-27.

11. Morgenstern EK. Interaction between genotype, site and silvicultural treatment. Can J For Res. 1982;3:329-332.

12. Paul AD, Foster GS, Caldwell T, McRae J. Trends in genetic and environment parameters for height, diameter, and volume in a multilocation clonal study with loblolly pine. For Sci. 1997;43:87-98. https://doi.org/10.1093/forestscience/43.1.87.

13. Bentzer BG, Foster GS, Hellberg AR, Podzorski AG. Genotype $\times$ environment interaction in Norway spruce involving three levels of genetic control: seed source, clone mixture, and clone. Can J For Res. 1988;18:11721181. https://doi.org/10.1139/x88-180.

14. Zhang PD, Wu F, Kang XY. Chemical properties of wood are under stronger genetic control than growth traits in Populus tomentosa Carr. Ann For Sci. 2015;72:89-97. https://doi.org/10.1007/s13595-014-0401-5.

15. Zhang PD, Wu F, Kang XY, Zhao CG, Li YJ. Genotypic Variations of Biomass Feedstock Properties for Energy in Triploid Hybrid Clones of Populus tomentosa. Bioenerg Res. 2015;8:1705-1713. https://doi.org/10.1007/s12155-015-9622-1.

16. Pliura A, Zhang SY, Mackay J, Bousquet J. Genotypic variation in wood density and growth traits of poplar hybrids at four clonal trials. For Ecol Manag. 2007;238:92-106. https://doi.org/10.1016/j.foreco.2006.09.082.

17. Zhang SY, Yu Q, Chauret G, Koubaa A. Selection for both growth and wood properties in hybrid poplar clones. For Sci. 2003:49:901-908. https://doi.org/10.1093/forestscience/49.6.901.

18. Mansfield SD, Weineisen H. Wood fiber quality and kraft pulping efficiencies of Trembling Aspen (Populus tremuloides Michx.) Clones. J Wood Chem Tech. 2007;27:135-151. https://doi.org/10.1080/02773810701700786.

19. Randall WK, Cooper DT. Predicted genotypic gains from cottonwood clonal tests. Silvae Genet. 1973;22:165167.

20. Lindgren D. Prediction and optimization of genetic gain with regard to genotype $\times$ environment interactions. 
Studia Forestalia Suecica. 1984;166:15-23.

21. Yu Q, Pulkkinen P, Rautio M, Haapanen M, Alen R, Stener LG, Beuker E, Tigerstedt PMA. Genetic control of wood physiochemical properties, growth and phenology in hybrid aspen clones. Can J For Res. 2001;31:13481356. https://doi.org/10.1139/x01-066.

22. Shukla GK. Some statistical aspects of partitioning genotype-environment components of variability. Hered. 1972;29:237-245. https://doi.org/10.1038/hdy.1972.87.

23. Finlay KW, Wilkinson GN. The analysis of adaptation in a plant breeding program. Aus $\mathbf{J}$ Agric Res. 1963;14:742-754. https://doi.org/10.1071/ar9630742.

24. Jain SK. Adaptive strategies: polymorphism, plasticity, and homeostasis. In: Solbrig, O.T., et al. (Eds.), Topics in Plant Population Biology. New York: Columbia University Press; 1979. p. 160-187.

25. Gillespie JH, Turelli M. Genotype-environment interactions and the maintenance of polygenic variation. Genetics. 1989;121:129-138.

26. Gouvêa LRL, Silva GAP, Verardi CK, Silva JQ, Scaloppi-Junior EJ, Gonçalves PDS. Temporal stability of vigor in rubber tree genotypes in the pre- and post-tapping phases using different methods. Euphytica. 2012;186: 625-634. https://doi.org/10.1007/s10681-012-0688-y.

27. Mohammadi RE, Amri A. Comparison of parametric and non-parametric methods for selecting stable and adapted durum wheat genotypes in variable environments. Euphytica. 2008;159:419-432. https://doi.org/10.1007/s10681-007-9600-6.

28. Kennedy RW. Fiber length of fast- and slow-grown black cottonwood. For Chron. 1957;33:46-50. https://doi.org/10.5558/tfc33046-1.

29. Posey CE, Bridgwater FE, Buxton JA. Natural variation in specific gravity, fiber length, and growth rate of eastern cottonwood in the southern Great Plains. Tappi. 1969;52:1508-1511.

30. Yanchuk AD, Dancik BP, Micko MM. Variation and heritability of wood density and fiber length of trembling aspen in Alberta, Canada. Silvae Genet. 1984;33:11-16.

31. Xie HF, Yu ZK, Chen DL, Guo DC, Song FX. Selection and genetic analysis for wood properties in pulpwood varieties of black poplar. J Shandong For Sci. 1996;1:1-5.

32. Wu F, Zhang PD, Pei JC, Kang XY. Genotypic parameters of wood density and fiber traits in triploid hybrid clones of Populus tomentosa at five clonal trials. Ann For Sci. 2013;70:751-759. https://doi.org/10.1007/s13595-013-0307-7.

33. Zhu ZT. Genetic improvement of Populus tomentosa. Beijing: Chinese Forestry Press; 2006. p. 155-220.

34. Chen ZS. The establishment of binomial stock volume tables of poplar. J For Res. 1989;2:78-83.

35. Becker WA. Manual of quantitative genetics. Washington: Academic Enterprises, Pullman; 1992. p. 160.

411 
Table 1 Location, climatic conditions, and description of six clonal trials

\begin{tabular}{ccccccc}
\hline Site & $\begin{array}{c}\text { Latitude } \\
\left({ }^{\circ} \mathrm{N}\right)\end{array}$ & $\begin{array}{c}\text { Longitude } \\
\left({ }^{\circ} \mathrm{W}\right)\end{array}$ & $\begin{array}{c}\text { Mean annual } \\
\mathrm{T}\left({ }^{\circ} \mathrm{C}\right)\end{array}$ & $\begin{array}{c}\text { Rainfall } \\
(\mathrm{mm} / \text { year })\end{array}$ & Number of clones & Sample trees \\
\hline Yanzhou & $35^{\circ} 10^{\prime}$ & $116^{\circ} 49^{\prime}$ & 13.6 & 660 & 10 & 54 \\
Gaotang & $36^{\circ} 51^{\prime}$ & $116^{\circ} 04^{\prime}$ & 13.1 & 556 & 10 & 54 \\
Xiangfen & $35^{\circ} 50^{\prime}$ & $111^{\circ} 21^{\prime}$ & 11.5 & 550 & 10 & 54 \\
Pinggu & $40^{\circ} 13^{\prime}$ & $117^{\circ} 12^{\prime}$ & 11.5 & 640 & 10 & 54 \\
Zhengzhou & $34^{\circ} 27^{\prime}$ & $113^{\circ} 57^{\prime}$ & 14.2 & 641 & 10 & 54 \\
Taiyuan & $37^{\circ} 52^{\prime}$ & $112^{\circ} 33^{\prime}$ & 10.5 & 431 & 10 & 54 \\
\hline
\end{tabular}

414

415

416

417

418

419

420

421

422

423

Table 2 Identity and origin of the hybrid clones

\begin{tabular}{|c|c|c|c|c|}
\hline No. & Clone identity & Parents & Level of ploidy & Sex \\
\hline 1 & B301 & $(P$. tomentosa $\times P$. bolleana $) \times P$. tomentosa & Triploid & q \\
\hline 2 & B302 & $(P$. tomentosa $\times P$. bolleana $) \times P$. tomentosa & Triploid & $0^{\lambda}$ \\
\hline 3 & B303 & $(P$. tomentosa $\times P$. bolleana $) \times P$. tomentosa & Triploid & q \\
\hline 4 & B304 & $(P$. tomentosa $\times P$. bolleana $) \times P$. tomentosa & Triploid & q \\
\hline 5 & B305 & $(P$. tomentosa $\times P$. bolleana $) \times P$. tomentosa & Triploid & q \\
\hline 6 & B306 & $(P$. tomentosa $\times P$. bolleana $) \times P$. tomentosa & Triploid & q \\
\hline 7 & B312 & $(P$. tomentosa $\times P$. bolleana $) \times P$. tomentosa & Triploid & $\hat{0}$ \\
\hline 8 & B330 & $(P$. alba $\times P$. glandulosa $) \times P$. tomentosa & Triploid & q \\
\hline 9 & B331 & $(P$. alba $\times P$. glandulosa $) \times P$. tomentosa & Triploid & q \\
\hline 10 & M1319 & P. tomentosa $\times P$. tomentosa & Diploid & $\hat{\sigma}$ \\
\hline
\end{tabular}


439 Table 3 Clone mean values, standard error (SE) of the mean, ranges of maximum and minimum values, and 440 coefficients of phenotypic variation ( $\mathrm{CV} \mathrm{p} \%)$ for fiber properties and growth traits at the six clonal trials.

\begin{tabular}{|c|c|c|c|c|c|c|}
\hline \multirow{2}{*}{ Site } & \multirow{2}{*}{ Traits } & \multicolumn{3}{|c|}{ Triploid clones } & \multicolumn{2}{|c|}{ Diploid clone (M1319) } \\
\hline & & Mean \pm SE & Range (min-max) & $\mathrm{CV} \mathrm{p} \%$ & Mean \pm SE & Range (min-max) \\
\hline \multirow{7}{*}{ Yanzhou } & $\mathrm{FL}(\mathrm{mm})$ & $0.77 \pm 0.04$ & $0.68 \pm 0.84$ & 5.3 & $0.67 \pm 0.01$ & $0.65 \pm 0.69$ \\
\hline & $\mathrm{FW}(\mu \mathrm{m})$ & $24.7 \pm 0.9$ & $23.2 \pm 26.6$ & 3.5 & $22.7 \pm 1.2$ & $21.9 \pm 25.0$ \\
\hline & FL/W & $31.3 \pm 1.4$ & $28.4 \pm 33.4$ & 4.5 & $29.4 \pm 1.1$ & $27.6 \pm 30.7$ \\
\hline & $\mathrm{C}(\mu \mathrm{g} / \mathrm{m})$ & $105.8 \pm 8.9$ & $86.5 \pm 124.3$ & 8.4 & $91.8 \pm 10.9$ & $83.3 \pm 113.4$ \\
\hline & $\mathrm{H}(\mathrm{m})$ & $12.5 \pm 1.2$ & $10.6 \pm 15.4$ & 9.7 & $9.9 \pm 0.5$ & $9.2 \pm 10.6$ \\
\hline & $\mathrm{DBH}(\mathrm{cm})$ & $10.7 \pm 1.0$ & $8.5 \pm 13.3$ & 9.4 & $9.0 \pm 1.2$ & $7.1 \pm 10.4$ \\
\hline & $\mathrm{SV}(\mathrm{dm} 3)$ & $48.2 \pm 11.0$ & $26.2 \pm 70.4$ & 22.9 & $28.7 \pm 8.2$ & $16.4 \pm 39.5$ \\
\hline \multirow{7}{*}{ Gaotang } & $\mathrm{FL}(\mathrm{mm})$ & $0.82 \pm 0.04$ & $0.73 \pm 0.90$ & 4.9 & $0.69 \pm 0.03$ & $0.64 \pm 0.74$ \\
\hline & $\mathrm{FW}(\mu \mathrm{m})$ & $24.2 \pm 0.9$ & $22.6 \pm 26.0$ & 3.6 & $22.1 \pm 0.5$ & $21.5 \pm 22.7$ \\
\hline & FL/W & $33.9 \pm 1.5$ & $30.7 \pm 37.1$ & 4.3 & $31.1 \pm 1.2$ & $29.7 \pm 32.6$ \\
\hline & $\mathrm{C}(\mu \mathrm{g} / \mathrm{m})$ & $102.2 \pm 7.4$ & $85.7 \pm 118.9$ & 7.3 & $85.5 \pm 3.0$ & $82.5 \pm 89.0$ \\
\hline & H (m) & $13.6 \pm 1.2$ & $9.4 \pm 16.0$ & 8.9 & $12.0 \pm 0.6$ & $11.1 \pm 12.9$ \\
\hline & $\mathrm{DBH}(\mathrm{cm})$ & $11.6 \pm 1.5$ & $7.8 \pm 14.9$ & 12.9 & $9.7 \pm 0.8$ & $8.6 \pm 10.8$ \\
\hline & $\mathrm{SV}(\mathrm{dm} 3)$ & $61.8 \pm 18.4$ & $23.6 \pm 113.9$ & 29.8 & $38.6 \pm 7.3$ & $28.1 \pm 48.9$ \\
\hline \multirow{7}{*}{ Xiangfen } & $\mathrm{FL}(\mathrm{mm})$ & $0.80 \pm 0.04$ & $0.73 \pm 0.90$ & 5.0 & $0.67 \pm 0.02$ & $0.64 \pm 0.71$ \\
\hline & $\mathrm{FW}(\mu \mathrm{m})$ & $24.2 \pm 0.9$ & $22.6 \pm 26.0$ & 3.7 & $22.0 \pm 0.7$ & $21.3 \pm 22.9$ \\
\hline & FL/W & $33.1 \pm 1.1$ & $30.3 \pm 35.4$ & 3.4 & $30.4 \pm 1.6$ & $28.1 \pm 32.7$ \\
\hline & $\mathrm{C}(\mu \mathrm{g} / \mathrm{m})$ & $99.6 \pm 9.3$ & $68.6 \pm 120.3$ & 9.3 & $82.0 \pm 5.7$ & $76.5 \pm 92.4$ \\
\hline & $\mathrm{H}(\mathrm{m})$ & $13.2 \pm 1.1$ & $11.4 \pm 15.8$ & 8.4 & $11.5 \pm 0.4$ & $10.8 \pm 12.1$ \\
\hline & $\mathrm{DBH}(\mathrm{cm})$ & $11.7 \pm 1.2$ & $9.0 \pm 14.6$ & 10.3 & $10.0 \pm 0.7$ & $8.8 \pm 10.7$ \\
\hline & $\mathrm{SV}(\mathrm{dm} 3)$ & $60.8 \pm 15.6$ & $31.5 \pm 107.1$ & 25.7 & $39.4 \pm 5.7$ & $28.8 \pm 46.4$ \\
\hline \multirow{7}{*}{ Pinggu } & $\mathrm{FL}(\mathrm{mm})$ & $0.76 \pm 0.04$ & $0.67 \pm 0.85$ & 5.3 & $0.61 \pm 0.02$ & $0.58 \pm 0.63$ \\
\hline & $\mathrm{FW}(\mu \mathrm{m})$ & $26.1 \pm 1.0$ & $23.7 \pm 28.8$ & 3.8 & $22.9 \pm 0.2$ & $22.5 \pm 23.1$ \\
\hline & FL/W & $29.1 \pm 1.6$ & $25.8 \pm 31.7$ & 5.5 & $26.5 \pm 0.9$ & $25.2 \pm 28.1$ \\
\hline & $\mathrm{C}(\mu \mathrm{g} / \mathrm{m})$ & $112.5 \pm 7.8$ & $97.8 \pm 129.4$ & 6.9 & $85.2 \pm 2.2$ & $80.9 \pm 87.2$ \\
\hline & H (m) & $11.4 \pm 1.5$ & $7.8 \pm 15.8$ & 13.2 & $10.6 \pm 0.9$ & $9.1 \pm 11.8$ \\
\hline & $\mathrm{DBH}(\mathrm{cm})$ & $13.8 \pm 2.2$ & $8.7 \pm 19.3$ & 15.9 & $10.4 \pm 1.3$ & $8.2 \pm 11.9$ \\
\hline & $\mathrm{SV}(\mathrm{dm} 3)$ & $75.3 \pm 28.0$ & $21.1 \pm 133.4$ & 37.2 & $39.8 \pm 10.6$ & $21.3 \pm 52.9$ \\
\hline \multirow{7}{*}{ Zhengzhou } & $\mathrm{FL}(\mathrm{mm})$ & $0.81 \pm 0.04$ & $0.74 \pm 0.89$ & 4.9 & $0.68 \pm 0.03$ & $0.64 \pm 0.72$ \\
\hline & $\mathrm{FW}(\mu \mathrm{m})$ & $25.3 \pm 0.8$ & $23.5 \pm 27.1$ & 3.2 & $22.2 \pm 0.6$ & $21.6 \pm 23.1$ \\
\hline & FL/W & $32.2 \pm 1.5$ & $28.8 \pm 34.8$ & 4.7 & $30.6 \pm 1.1$ & $29.1 \pm 32.2$ \\
\hline & $\mathrm{C}(\mu \mathrm{g} / \mathrm{m})$ & $111.2 \pm 8.6$ & $88.2 \pm 127.5$ & 7.7 & $87.5 \pm 3.8$ & $82.3 \pm 91.4$ \\
\hline & $\mathrm{H}(\mathrm{m})$ & $13.0 \pm 1.5$ & $9.2 \pm 15.1$ & 11.5 & $11.1 \pm 0.8$ & $10.4 \pm 12.6$ \\
\hline & $\mathrm{DBH}(\mathrm{cm})$ & $14.4 \pm 1.8$ & $9.8 \pm 18.5$ & 12.5 & $12.3 \pm 1.0$ & $11.1 \pm 13.6$ \\
\hline & $\mathrm{SV}(\mathrm{dm} 3)$ & $91.0 \pm 26.7$ & $32.9 \pm 152.7$ & 29.3 & $57.2 \pm 12.2$ & $44.3 \pm 75.5$ \\
\hline \multirow{7}{*}{ Taiyuan } & $\mathrm{FL}(\mathrm{mm})$ & $0.78 \pm 0.04$ & $0.72 \pm 0.87$ & 5.1 & $0.65 \pm 0.03$ & $0.61 \pm 0.68$ \\
\hline & $\mathrm{FW}(\mu \mathrm{m})$ & $24.6 \pm 0.9$ & $22.6 \pm 27.4$ & 3.7 & $21.6 \pm 0.4$ & $21.0 \pm 22.1$ \\
\hline & FL/W & $31.7 \pm 1.1$ & $29.0 \pm 34.2$ & 3.5 & $29.9 \pm 1.4$ & $27.9 \pm 31.4$ \\
\hline & $\mathrm{C}(\mu \mathrm{g} / \mathrm{m})$ & $103.5 \pm 8.4$ & $84.4 \pm 120.0$ & 8.1 & $80.0 \pm 1.6$ & $77.7 \pm 81.8$ \\
\hline & $\mathrm{H}(\mathrm{m})$ & $13.9 \pm 1.7$ & $9.5 \pm 16.4$ & 12.2 & $11.7 \pm 0.5$ & $11.0 \pm 12.2$ \\
\hline & $\mathrm{DBH}(\mathrm{cm})$ & $14.8 \pm 1.9$ & $11.3 \pm 18.5$ & 12.8 & $13.0 \pm 0.8$ & $11.7 \pm 13.6$ \\
\hline & $\mathrm{SV}(\mathrm{dm} 3)$ & $100.9 \pm 31.1$ & $46.1 \pm 172.4$ & 30.8 & $65.5 \pm 9.2$ & $50.7 \pm 74.6$ \\
\hline
\end{tabular}

441

442

443 
444

445

446

Table 4 Analysis of variance results, estimates of repeatability $( \pm \mathrm{SE})$ at the clonal mean and individual-tree levels of all traits of the nine triploid hybrid clones at the six sites combined

\begin{tabular}{|c|c|c|c|c|c|c|}
\hline \multirow{2}{*}{ Traits } & \multicolumn{3}{|c|}{ P-value } & \multirow{2}{*}{$R_{b}^{2}$} & \multirow{2}{*}{$R_{c}^{2}$} & \multirow{2}{*}{ Percentage (\%) } \\
\hline & Clones & Sites & Sites $\times$ clones & & & \\
\hline FL & 0.000 & 0.000 & 0.000 & $0.29 \pm 0.11$ & $0.89 \pm 0.05$ & 73.9 \\
\hline FW & 0.000 & 0.000 & 0.031 & $0.36 \pm 0.13$ & $0.93 \pm 0.03$ & 87.6 \\
\hline FL/W & 0.000 & 0.000 & 0.014 & $0.17 \pm 0.08$ & $0.83 \pm 0.07$ & 68.4 \\
\hline $\mathrm{C}$ & 0.000 & 0.000 & 0.000 & $0.35 \pm 0.13$ & $0.91 \pm 0.04$ & 72.7 \\
\hline $\mathrm{H}$ & 0.000 & 0.000 & 0.001 & $0.18 \pm 0.09$ & $0.76 \pm 0.09$ & 42.1 \\
\hline DBH & 0.000 & 0.000 & 0.001 & $0.21 \pm 0.10$ & $0.85 \pm 0.06$ & 66.2 \\
\hline SV & 0.000 & 0.000 & 0.000 & $0.19 \pm 0.09$ & $0.80 \pm 0.08$ & 52.9 \\
\hline
\end{tabular}

Note: Estimated percentage ratio (\%) of clonal variance component to the total variance component of clonexsite interaction and clone. The degrees of freedom are 5 for the site, 8 for the clone, 40 for the clone $\times$ interaction, and 270 for the error

Table 5 The results of analysis of variance and estimates of repeatability of all studied traits of the nine triploid hybrid clones at individual site

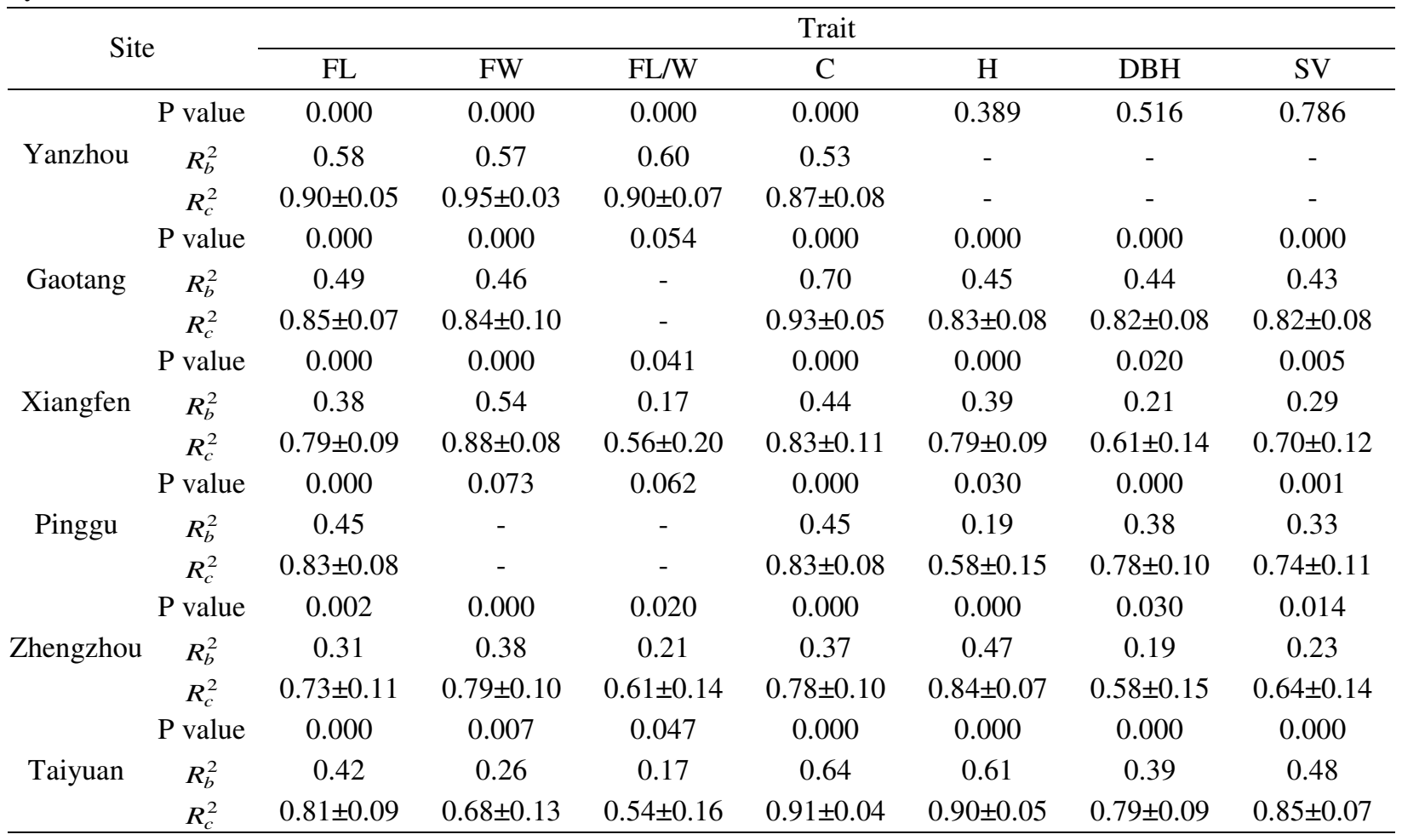

455

456

457

458

459

460

461 
Table 6 The estimates of type B genetic correlations among all possible pairs of sites for fiber properties and growth traits (with approximate SE in brackets) of the nine triploid hybrid clones

\begin{tabular}{cccccccc}
\hline \multirow{2}{*}{ Pairs of site } & \multicolumn{7}{c}{ Genetic correlation } \\
\cline { 2 - 8 } & $\mathrm{FL}$ & $\mathrm{FW}$ & $\mathrm{FL} / \mathrm{W}$ & $\mathrm{C}$ & $\mathrm{H}$ & $\mathrm{DBH}$ & $\mathrm{SV}$ \\
\hline YZ-GT & $0.91(0.01)^{*}$ & $0.97(0.01)^{*}$ & - & $0.63(0.03)$ & - & - & - \\
YZ-XF & $0.90(0.01)^{*}$ & $0.59(0.02)$ & $0.67(0.03)$ & $0.58(0.05)$ & - & - & - \\
YZ-PG & $0.92(0.01)^{*}$ & - & - & $0.91(0.01)^{*}$ & - & - & - \\
YZ-ZZ & $0.68(0.04)$ & $0.86(0.01)^{*}$ & $1.13(0.01)^{* *}$ & $0.70(0.02)$ & - & - & - \\
YZ-TY & $0.12(0.05)$ & $1.01(0.01)^{* *}$ & $0.20(0.09)$ & $0.54(0.02)$ & - & - & - \\
GT-XF & $0.98(0.01)^{*}$ & $0.90(0.01)^{*}$ & - & $0.55(0.04)$ & $0.69(0.03)$ & $0.57(0.07)$ & $0.51(0.07)$ \\
GT-PG & $0.99(0.01)^{*}$ & - & - & $0.74(0.01)$ & $1.32(0.04)^{* *}$ & $0.96(0.01)^{*}$ & $0.98(0.01)^{*}$ \\
GT-ZZ & $0.91(0.01)^{*}$ & $0.96(0.01)^{*}$ & - & $0.67(0.02)$ & $0.88(0.01)^{*}$ & $1.13(0.02)^{* *}$ & $1.01(0.01)^{* *}$ \\
GT-TY & $0.35(0.06)$ & $1.03(0.01)^{* *}$ & - & $0.66(0.01)$ & $0.16(0.04)$ & $0.61(0.03)$ & $0.44(0.04)$ \\
XF-PG & $0.90(0.01)^{*}$ & - & - & $1.05(0.01)^{* *}$ & $1.06(0.01)^{* *}$ & $0.91(0.01)^{*}$ & $0.84(0.02)$ \\
XF-ZZ & $1.05(0.01)^{* *}$ & $0.79(0.02)$ & $0.84(0.03)$ & $1.16(0.01)^{* *}$ & $0.79(0.02)$ & $1.25(0.04)^{* *}$ & $1.08(0.01)^{* *}$ \\
XF-TY & $0.26(0.07)$ & $0.56(0.04)$ & $0.01(0.01)$ & $0.70(0.02)$ & $0.45(0.03)$ & $0.22(0.09)$ & $0.19(0.07)$ \\
PG-ZZ & $0.96(0.01)^{*}$ & - & - & $1.10(0.01)^{* *}$ & $1.13(0.01)^{* *}$ & $1.27(0.03)^{* *}$ & $1.26(0.03)^{* *}$ \\
PG-TY & $0.08(0.07)$ & - & - & $0.68(0.02)$ & $0.55(0.04)$ & $0.66(0.03)$ & $0.60(0.03)$ \\
ZZ-TY & $0.28(0.08)$ & $0.96(0.01)^{*}$ & $0.64(0.07)$ & $0.64(0.02)$ & $-0.02(0.01)$ & $0.59(0.05)$ & $0.36(0.06)$ \\
\hline
\end{tabular}

470

471

472

473

474

475

476

477

478

479

480

481

482

483

484

485

486

487

488

489

490

Note: $*$ Significant correlations $p<0.05$. $* *$ denotes $p<0.01$ 
491

492

493

494

495

496

497

498

499

500

501

Table 7 Stability parameters for FL and SV of the nine triploid hybrid clones across six sites

\begin{tabular}{|c|c|c|c|c|c|c|c|c|}
\hline \multirow{2}{*}{ Traits } & \multirow{2}{*}{ Clones } & \multicolumn{3}{|c|}{ Shukla stability variance } & \multicolumn{4}{|c|}{ Finlay-Wilkinson stability parameters } \\
\hline & & Variance $\times 10^{-4}$ & $F$ & $P$ & Intercept & $t$ & $b i$ & $t$ \\
\hline \multirow{9}{*}{ FL } & B301 & 4.31 & 3.14 & 0.009 & 0.068 & 0.22 & 0.929 & 2.41 \\
\hline & B302 & 4.56 & 3.33 & 0.006 & -0.241 & -0.91 & 1.321 & 3.97 \\
\hline & B303 & 3.80 & 2.77 & 0.018 & -0.188 & -0.68 & 1.214 & 3.49 \\
\hline & B304 & 4.06 & 2.96 & 0.013 & -0.204 & -0.74 & 1.250 & 3.59 \\
\hline & B305 & 6.34 & 4.62 & 0.000 & 0.043 & 0.12 & 0.893 & 1.91 \\
\hline & B306 & 6.59 & 4.81 & 0.000 & -0.315 & -0.94 & 1.429 & 3.37 \\
\hline & B312 & 5.83 & 4.25 & 0.001 & 0.193 & 0.58 & 0.750 & 1.79 \\
\hline & B330 & 1.01 & 0.74 & 0.594 & 0.115 & 0.80 & 0.893 & 4.93 \\
\hline & B331 & 2.28 & 1.66 & 0.143 & 0.246 & 1.35 & 0.679 & 2.95 \\
\hline \multirow{9}{*}{ SV } & B301 & 3.16 & 5.39 & 0.000 & 18.673 & 0.78 & 0.667 & 2.10 \\
\hline & B302 & 0.56 & 0.95 & 0.448 & 11.26 & 0.73 & 0.870 & 4.27 \\
\hline & B303 & 0.95 & 1.63 & 0.153 & 13.233 & 0.83 & 0.908 & 4.28 \\
\hline & B304 & 2.43 & 4.14 & 0.001 & -25.668 & -1.11 & 1.315 & 4.26 \\
\hline & B305 & 2.02 & 3.44 & 0.005 & 14.072 & 1.12 & 0.466 & 2.79 \\
\hline & B306 & 0.72 & 1.22 & 0.298 & 0.923 & 0.07 & 0.941 & 5.07 \\
\hline & B312 & 1.23 & 2.11 & 0.065 & 13.508 & 0.74 & 0.919 & 3.78 \\
\hline & B330 & 2.12 & 3.61 & 0.004 & -26.084 & -1.39 & 1.411 & 5.64 \\
\hline & B331 & 2.04 & 3.48 & 0.005 & -19.436 & -1.22 & 1.477 & 7.00 \\
\hline
\end{tabular}

502 Note: $F$ : Fisher's criteria; $P$ : probability levels; $t$ : Student's criteria; $b_{i}$ : regression coefficient

503

504

505

506

507

508

509

510

511

512

513

514

515

516

517 
Table 8 Phenotypic correlations (below the diagonal) and genotypic correlations (above the diagonal) among all 532 traits at individual sites of the nine triploid hybrid clones

\begin{tabular}{|c|c|c|c|c|c|c|c|c|}
\hline Site & Traits & FL & FW & FL/W & $\mathrm{C}$ & $\mathrm{H}$ & DBH & SV \\
\hline \multirow{7}{*}{ Yanzhou } & FL & & 0.59 & $0.77 *$ & $0.87 *$ & - & - & - \\
\hline & FW & 0.55 & & -0.25 & $0.97 *$ & - & - & - \\
\hline & FL/W & $0.69^{*}$ & -0.23 & & 0.16 & - & - & - \\
\hline & $\mathrm{C}$ & $0.77 *$ & $0.88^{*}$ & 0.14 & & - & - & - \\
\hline & $\mathrm{H}$ & 0.10 & -0.14 & 0.24 & -0.29 & & - & - \\
\hline & $\mathrm{DBH}$ & 0.26 & -0.34 & 0.59 & -0.05 & 0.24 & & - \\
\hline & SV & 0.29 & -0.49 & $0.75^{*}$ & -0.12 & $0.74 *$ & $0.90 * *$ & \\
\hline \multirow{8}{*}{ Gaotang } & FL & & $0.92 *$ & - & 0.71 & 0.65 & 0.32 & 0.35 \\
\hline & FW & $0.78 *$ & & - & $0.99 * *$ & 0.25 & -0.19 & -0.16 \\
\hline & FL/W & $0.68 *$ & 0.08 & & - & - & - & - \\
\hline & $\mathrm{C}$ & 0.63 & $0.88 * *$ & 0.04 & & -0.01 & -0.34 & -0.30 \\
\hline & $\mathrm{H}$ & 0.55 & 0.21 & 0.64 & -0.01 & & $0.82 *$ & $0.93 *$ \\
\hline & $\mathrm{DBH}$ & 0.27 & -0.16 & 0.60 & -0.30 & $0.68 *$ & & $1.20 * *$ \\
\hline & SV & 0.29 & -0.13 & 0.61 & -0.26 & $0.77 *$ & $0.98 * *$ & \\
\hline & FL & & $0.96 * *$ & 0.63 & $0.84^{*}$ & 0.52 & 0.66 & 0.56 \\
\hline \multirow{6}{*}{ Xiangfen } & FW & $0.80 * *$ & & -0.27 & $0.96^{* *}$ & -0.01 & 0.07 & 0.03 \\
\hline & FL/W & 0.42 & -0.19 & & -0.19 & $1.04 *$ & $1.16^{*}$ & $1.03^{*}$ \\
\hline & $\mathrm{C}$ & $0.68 *$ & $0.82 * *$ & -0.13 & & -0.32 & -0.11 & -0.21 \\
\hline & $\mathrm{H}$ & 0.41 & -0.01 & $0.69^{*}$ & -0.26 & & $1.37 * *$ & $1.31^{* *}$ \\
\hline & DBH & 0.46 & 0.05 & $0.68^{*}$ & -0.08 & $0.95 * *$ & & $1.51^{* *}$ \\
\hline & SV & 0.42 & 0.02 & 0.65 & -0.16 & $0.98 * *$ & $0.99 * *$ & \\
\hline \multirow{7}{*}{ Pinggu } & FL & & - & - & $0.75^{*}$ & $1.10^{* *}$ & 0.56 & 0.62 \\
\hline & FW & 0.49 & & - & - & - & - & - \\
\hline & FL/W & $0.81 * *$ & -0.11 & & - & - & - & - \\
\hline & $\mathrm{C}$ & $0.68^{*}$ & $0.86^{* *}$ & 0.20 & & 0.40 & -0.19 & -0.16 \\
\hline & $\mathrm{H}$ & $0.88 * *$ & 0.17 & $0.90^{* *}$ & 0.31 & & $1.05^{* *}$ & $1.11^{* *}$ \\
\hline & DBH & 0.51 & -0.25 & $0.80 *$ & -0.18 & $0.80 * *$ & & $1.16^{* *}$ \\
\hline & SV & 0.54 & -0.24 & $0.83^{* *}$ & -0.15 & $0.83^{* *}$ & $0.99 * *$ & \\
\hline \multirow{6}{*}{ Zhengzhou } & FL & & 0.63 & $0.89 *$ & 0.68 & 0.49 & 0.46 & 0.43 \\
\hline & FW & 0.54 & & -0.33 & $0.93 * *$ & -0.23 & -0.52 & -0.53 \\
\hline & FL/W & $0.68 *$ & -0.26 & & -0.06 & $0.85^{*}$ & $1.10^{*}$ & $1.07 *$ \\
\hline & C & 0.58 & $0.82 * *$ & -0.05 & & 0.29 & -0.20 & -0.10 \\
\hline & $\mathrm{H}$ & 0.44 & -0.21 & $0.69^{*}$ & 0.26 & & $0.97 *$ & $1.08 * *$ \\
\hline & $\mathrm{DBH}$ & 0.34 & -0.40 & $0.74 *$ & -0.15 & $0.76^{*}$ & & $1.42 * *$ \\
\hline
\end{tabular}




\begin{tabular}{ccccccccc} 
& SV & 0.34 & -0.42 & $0.75^{*}$ & -0.08 & $0.88^{* *}$ & $0.97 * *$ & \\
& FL & & $0.97 * *$ & $1.01^{*}$ & $0.83^{* *}$ & 0.33 & 0.24 & 0.34 \\
& FW & $0.82^{* *}$ & & 0.36 & $1.01 * *$ & 0.17 & -0.28 & -0.11 \\
& FL/W & $0.76^{*}$ & 0.25 & & 0.43 & 0.47 & 0.84 & 0.83 \\
& $\mathrm{C}$ & $0.82 * *$ & $0.91 * *$ & 0.34 & & -0.05 & -0.23 & -0.15 \\
& $\mathrm{H}$ & 0.32 & 0.15 & 0.37 & -0.05 & & 0.67 & $0.82^{*}$ \\
& $\mathrm{DBH}$ & 0.21 & -0.23 & 0.61 & -0.22 & 0.62 & & $1.09 * *$ \\
& $\mathrm{SV}$ & 0.31 & -0.09 & 0.63 & -0.15 & $0.79 *$ & $0.97 * *$ & \\
\hline
\end{tabular}

533

534

535

536

537

538

539

540

541

542

543

544

36

38

50

52

Note: *Significant correlations $=0.01<p<0.05 ; * *$ denotes $p<0.01$

Note: Significant correlations $=0.01<p<0.05 ;$ " denotes $p<0.01$

Table 9 Expected response $(\Delta G / \bar{X} \times 100)$ in fiber properties and growth traits at the six sites for different selection criteria of the nine triploid hybrid clones

\begin{tabular}{lcccccccc}
\hline \multirow{2}{*}{ Selection criterion } & \multicolumn{7}{c}{ Response (\%) } \\
\cline { 3 - 8 } Yanzhou & FL & FW & FL/W & C & H & DBH & SV \\
& FL & $\mathbf{4 . 9}$ & 2.2 & 3.3 & 6.6 & - & - & - \\
& FW & 3.6 & $\mathbf{3 . 9}$ & -1.1 & 7.5 & - & - & - \\
& FL/W & 3.9 & -0.9 & $\mathbf{4 . 3}$ & 1.2 & - & - & - \\
& C & 4.2 & 3.6 & 0.7 & $\mathbf{7 . 5}$ & - & - & - \\
& FL & $\mathbf{4 . 1}$ & 2.7 & - & 5.2 & 3.7 & 6.6 & 13.7 \\
& FW & 3.8 & $\mathbf{3 . 0}$ & - & 7.3 & 1.8 & -1.9 & -3.9 \\
& FL/W & - & - & - & - & - & - & - \\
Gaotang & C & 3.1 & 3.1 & - & $\mathbf{7 . 8}$ & -0.1 & -3.5 & -7.7 \\
& H & 2.3 & 0.7 & - & -0.1 & $\mathbf{7 . 0}$ & 6.7 & 17.6 \\
& DBH & 1.1 & -0.6 & - & -2.5 & 4.7 & $\mathbf{9 . 8}$ & 22.3 \\
& SV & 1.2 & -0.5 & - & -2.2 & 5.3 & 9.6 & $\mathbf{2 2 . 7}$ \\
& FL & $\mathbf{3 . 2}$ & 3.0 & 1.0 & 6.0 & 3.0 & 3.4 & 13.4 \\
& FW & 3.2 & $\mathbf{3 . 3}$ & -0.5 & 7.2 & -0.1 & 0.4 & 0.8 \\
& FL/W & 1.7 & -0.7 & $\mathbf{1 . 4}$ & -1.1 & 5.1 & 4.4 & 10.8 \\
& C & 2.7 & 3.1 & -0.3 & $\mathbf{7 . 4}$ & -1.9 & -0.6 & -5.2 \\
& H & 1.3 & -0.1 & 1.6 & -2.2 & $\mathbf{5 . 8}$ & 4.6 & 13.5 \\
& DBH & 1.3 & 0.2 & 1.4 & -0.7 & 4.8 & $\mathbf{4 . 7}$ & 14.9 \\
& SV & 1.4 & 0.1 & 1.6 & -1.7 & 5.3 & 4.5 & $\mathbf{1 5 . 4}$ \\
& FL & $\mathbf{3 . 8}$ & - & - & 4.6 & 7.9 & 9.0 & 17.8
\end{tabular}




\begin{tabular}{|c|c|c|c|c|c|c|c|c|}
\hline \multirow{7}{*}{ Zhengzhou } & FL & 3.3 & 1.6 & 2.6 & 4.0 & 4.4 & 4.0 & 8.4 \\
\hline & FW & 2.2 & 2.6 & -1.0 & 5.7 & -2.1 & -4.6 & -10.6 \\
\hline & FL/W & 2.7 & -0.8 & 2.7 & -0.3 & 7.0 & 8.7 & 19.0 \\
\hline & $\mathrm{C}$ & 2.3 & 2.4 & -0.2 & 6.1 & 2.7 & -1.8 & -2.0 \\
\hline & $\mathrm{H}$ & 1.8 & -0.6 & 2.7 & 1.8 & 9.6 & 9.1 & 22.4 \\
\hline & $\mathrm{DBH}$ & 1.4 & -1.2 & 2.9 & -1.0 & 7.8 & 7.8 & 24.6 \\
\hline & SV & 1.4 & -1.2 & 2.9 & -0.6 & 9.0 & 11.5 & 18.1 \\
\hline \multirow{7}{*}{ Taiyuan } & FL & 4.0 & 2.6 & 2.3 & 6.6 & 3.7 & 2.7 & 9.3 \\
\hline & $\mathrm{FW}$ & 3.5 & 2.4 & 0.7 & 7.4 & 1.7 & -2.9 & -2.7 \\
\hline & FL/W & 3.3 & 0.8 & 1.9 & 2.8 & 4.3 & 7.9 & 18.8 \\
\hline & $\mathrm{C}$ & 3.5 & 2.8 & 1.0 & 8.4 & -0.6 & -2.8 & -4.5 \\
\hline & $\mathrm{H}$ & 1.4 & 0.5 & 1.1 & -0.4 & 11.9 & 8.1 & 24.0 \\
\hline & DBH & 0.9 & -0.7 & 1.9 & -1.8 & 7.4 & 11.3 & 29.6 \\
\hline & SV & 1.4 & -0.3 & 1.9 & -1.3 & 9.5 & 12.7 & 28.2 \\
\hline
\end{tabular}

546 
Figures
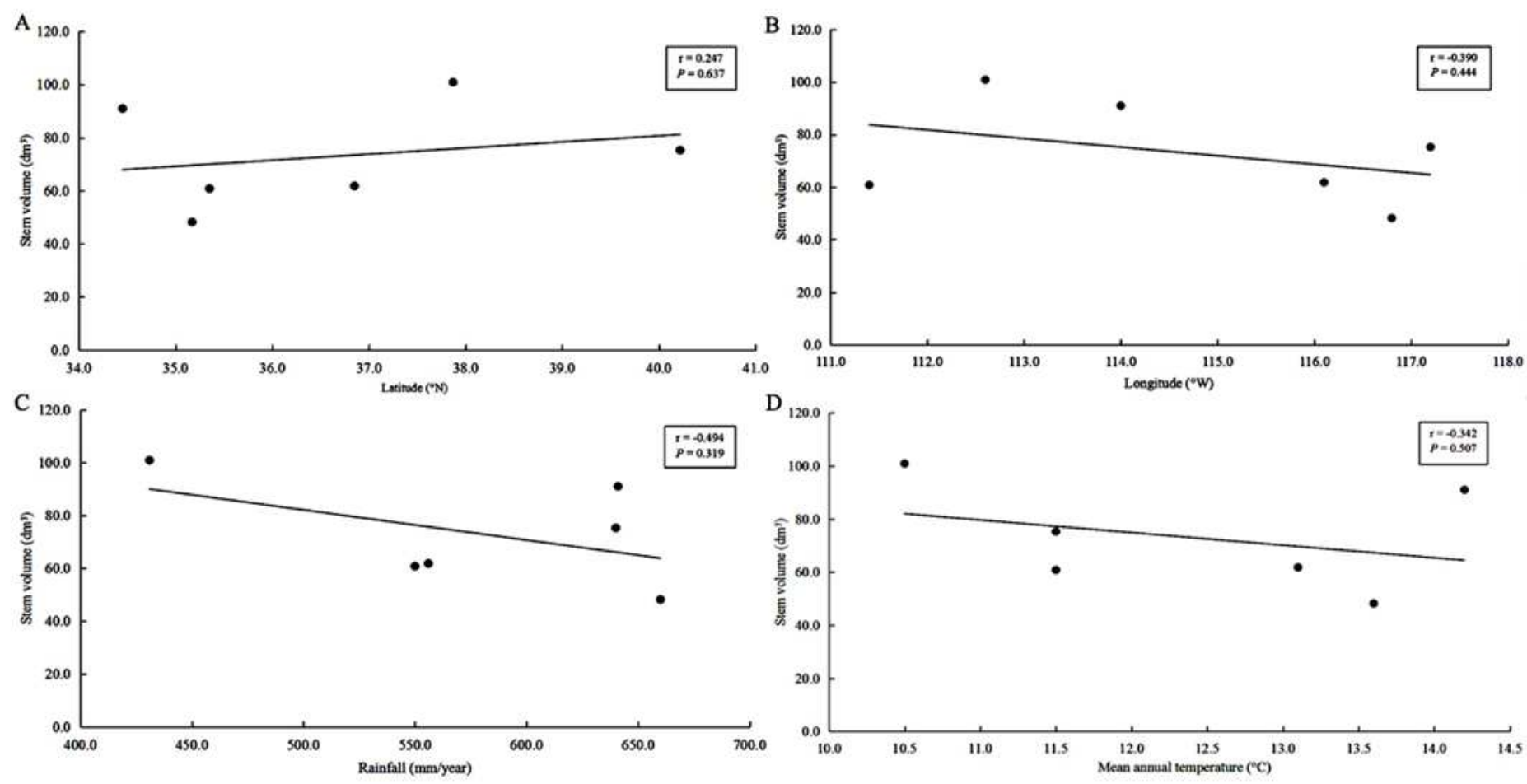

\section{Figure 1}

Correlations between stem volume and (A) latitude, (B) longitude, (C) rainfall, and (D) mean annual temperature 